\title{
Research on Application of SDN in the New Generation Power Dispatching Data Network
}

\author{
Guiying Zhu', a , Jihao $\mathrm{Hu}^{2}$, Zijian Yan ${ }^{1}$,Shuiping $\mathrm{Liu}^{3}$, Xiaomei Liu ${ }^{1}$, Peng Sun ${ }^{1}$ \\ and Hong Zhang ${ }^{1}$ \\ ${ }^{1}$ Power Automation Research Institute, China Electric Power Research Institute, Nanjing, China \\ ${ }^{2}$ NARI Solar Energy Technology Co. Ltd, Nanjing, China \\ ${ }^{3}$ Xiushui County New Rural Power Supply Service Co. Ltd, Jiangxi, China \\ azhuguiying@epri.sgcc.com.cn
}

Keywords: SDN, power dispatching data network, new generation.

Abstract. Along with the rapid development of UHV AC/DC interconnected power grid and the construction of the power dispatching data network, the resources allocation capability of power grid in wide area has been enhanced greatly. Now, the power grid operation faces a series of challenges, such as the centralized monitoring level is not high enough, the global decision-making ability is insufficient, dispatching a wide range of ability to optimize the allocation of resources is shortage and lacks of absorptive capacity of new energy. This paper analyzes the demands for SDN of the dispatching data network, discusses the application of the key technology in the new generation power dispatching data network, sets up the experimental environment to verify the adaptability of SDN technology in the new generation power dispatching data network.

\section{Introduction}

In recent years, software defined network (SDN) technology has become one of the hottest new network technology ${ }^{[1-4]}$, it is the liberation of manual operation, reducing configuration error, quick and easy to deploy network environment, and has been successfully applied in the network in the data center and cloud computing. However, in the power communication network, the application of SDN is still in early stage. In view of the contention of the SDN technology itself and the specific characteristics of power business, it is very necessary to argue the adaptability of SDN technology in electric power communication networks.

In 2013 , the company of State Power Grid launched a project that is named "research on the overall framework of new generation power dispatching technical support system", which puts forward the framework of "physical distribution architecture, logical unity", establishes the overall mechanism of real-time data processing and storage. The traffic of the dispatching data network is much larger than before. On the other hand, AGC analysis results in Senior Application Decision Center need to be sent to the dispatching center, which regulate the generator output of the corresponding plant. The transmission of control information cannot be delayed. Otherwise it will affect the result of regulation and the state of power grid operation. In addition, the future design of dispatching system proposed that communication between substation and the master station is based on broadband internet thinking and is service oriented. All these things break the traditional mode of substation and master station connecting, which can realize the wide area backup on master, substation as a node on the network, can provide data for other master, but the reliability of the network needs to be safeguarded. These are the new challenges for dispatching data network. The paper [5] [6] [7] discussed the application of SDN technology in power data center and in power communication network, but they didn't discuss the application in power dispatching data network. This paper will analyze the demand of application of SDN technology in power dispatching data network and the key technology, and verify the adaptability of SDN technology in the new generation power dispatching data network. 


\section{Requirement Analysis of the Power Dispatching Data Network}

The future dispatching system based on the "physical distribution, unified logic" put forward higher requirements for the wide area network resource management and data access, especially it is necessary to ensure the timeliness of the control interaction information in a wide area. Therefore, that put forward a very high demand on the transmission of the dispatching data network. It is mainly reflected in the following 4 aspects.

\section{It is difficult to support the frequent access to the wide area data on the traditional network.}

Considering the accuracy analysis of application, the future dispatching system is established with a large and complete model. The integrated analysis and decision center needs to obtain real-time data from all levels of dispatching center through wide area network, that results the data is much larger than before and puts forward higher requirements on the bandwidth of the network. The equipment in traditional network use hop by hop routing algorithm, that lack of global view, cannot grasp the real-time operational status of the network and cannot achieve the global dispatching network source, so that cannot ensure the communication of the key business. SDN separates the control layer from the data layer, and can get the global view of the network, so that it can allocate and optimize network resources globally. For the exception of the situation, it also can be quickly restored.

\section{It is difficult to ensure the timely and reliable transmission of information transmission in power grid control.}

The existing dispatching data network does not have a clear message level division for the control class. It is hybrid transmission of high priority control message with low priority non control message. Existing network can only support business classification and quality control by VPN.

In the center of integrated analysis and decision, the control command of AGC/AVC will be issued through the wide area network. This kind of control message is very important, different from other ordinary message, maybe be blocked in the future along with business growth. So it is necessary to ensure that important message bandwidth is not affected. The traditional network also has the function of flow control, but the configuration is more complex, need to configure each device. If a new business needs QoS flow control, it is a huge workload. Intelligent SDN network were realized by software, can well classify all kinds of messages, customize various network parameters, such as routing, security, strategy, QoS, traffic engineering.

\section{The mode of substation network access in future will bring challenges to network reliability.}

The traditional substation is access to the master station in private line, the main disadvantages are: (1) when the substation is access to another master station, it need to build a another line, the price is relatively high; (2) when there is a problem with the master station, the other master station is unable to receive the data of the substation connected with the fault master station, so cannot take over the substation. In the design of future dispatching system, substation is based on the broadband Internet thinking, with the network mode to access the data network. As a node in a data network, any authorized master can access to the substation data. Through the SDN network, it can achieve for the substation plug and play. After decoupling SDN control plane and data plane, it is only need to configure the controller and the other network devices do not need modification, all links are automatically accomplished by the controller, greatly reducing the workload of network configuration.

\section{Network Architecture}

The traditional power dispatching data network adopts the hierarchical network structure, which includes backbone network and access network. The backbone network is consist of State dispatching network, branch dispatching network, province dispatching network and area dispatching network. 
The direct dispatching substations of dispatching center at all levels consist of the access network, such as state dispatching access network, branch dispatching access network, province dispatching access network and area dispatching access network. The network structure of the dispatching data network based on SDN is divided into two planes of control and forward, so that the two planes can be independent and flexible. The architecture of dispatching data network based on SDN is shown in Fig. 1. The forwarding plane still uses a layered architecture, and at the same time, the controller is added to the network control plane.

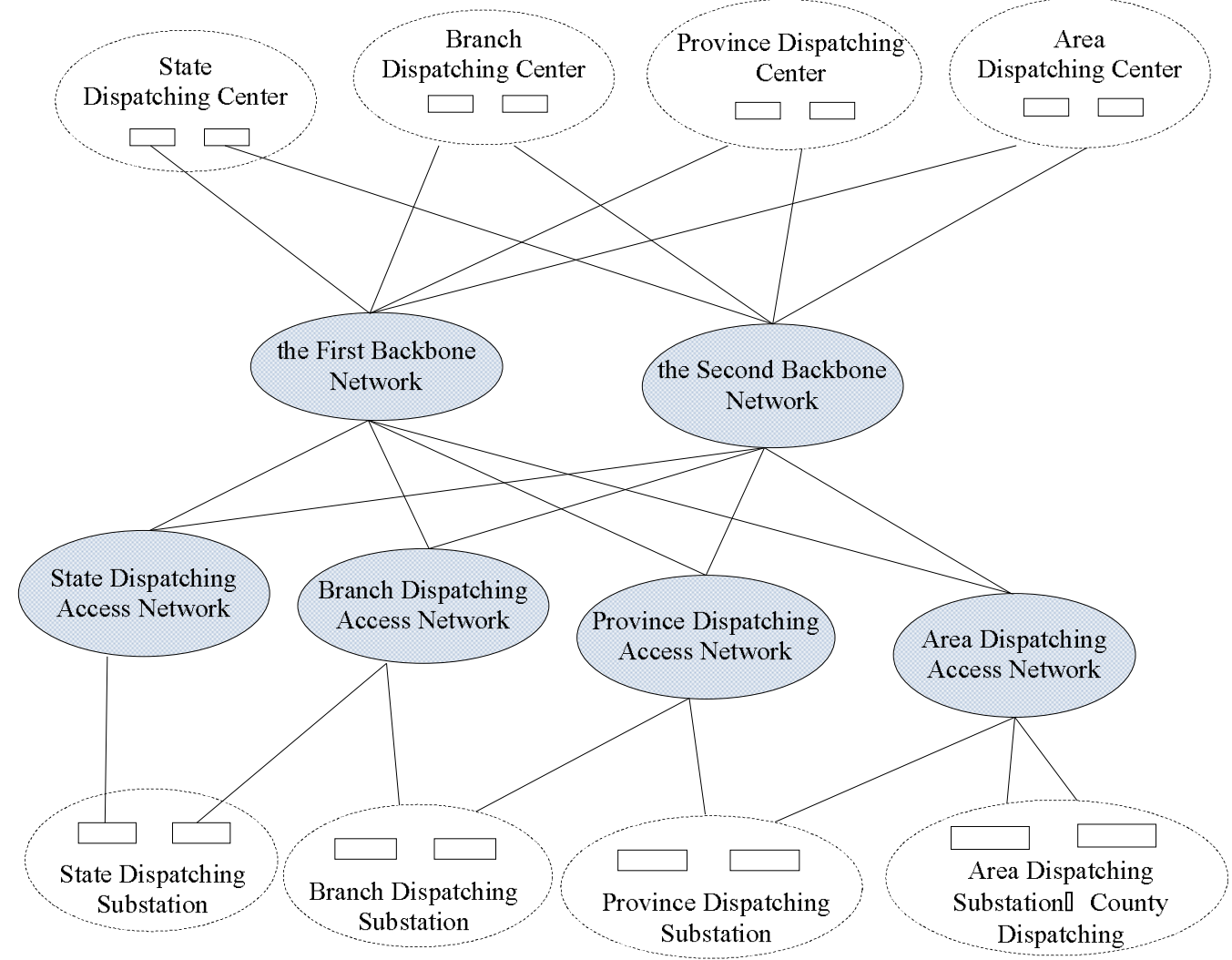

Fig. 1 dispatching data network architecture based on SDN

The deployment diagram of the control plane is shown in Fig. 2.

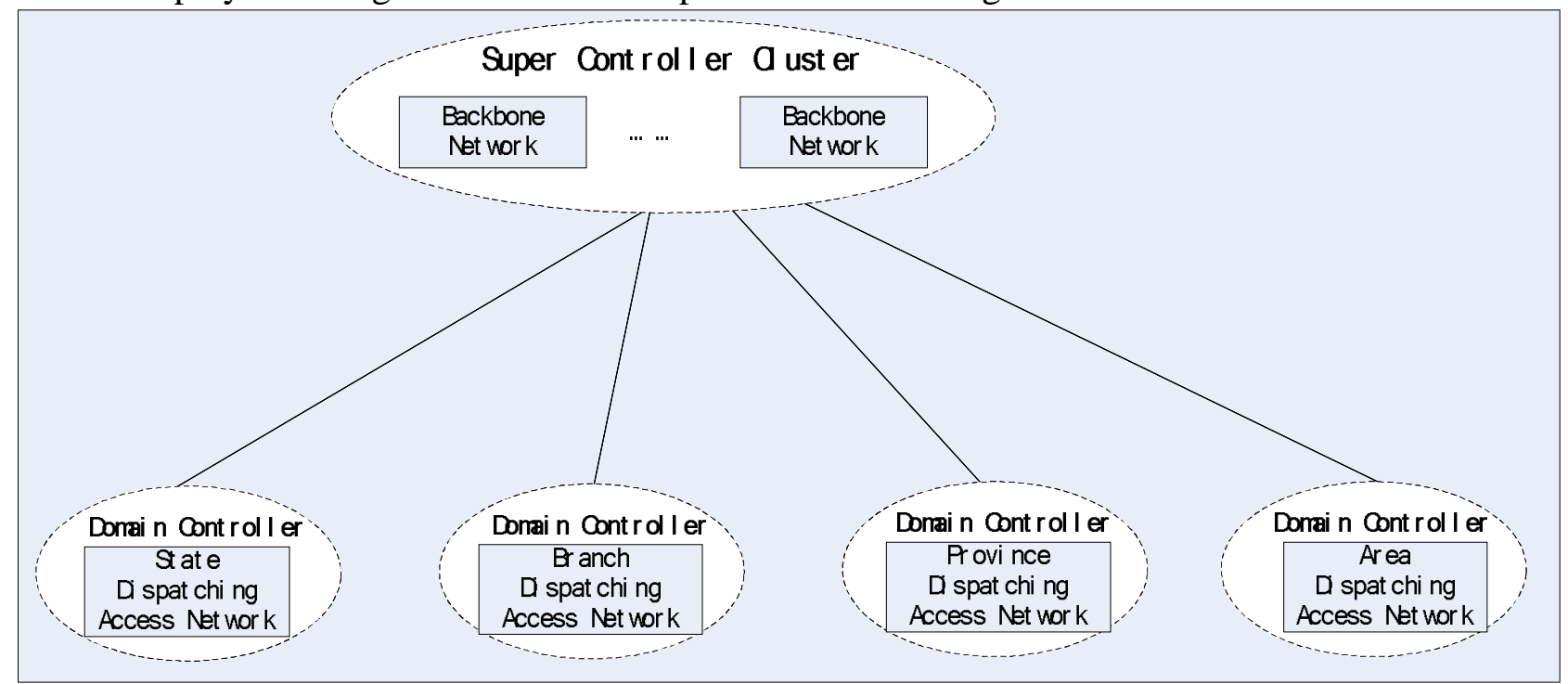

Fig. 2 the deployment diagram of the control plane

The controllers are deployed according to the autonomous domain, every domain has a controller, which is called domain controller. Then deploy another controller on top of domain controller, which is a cluster. The top controller is called super controller or parent controller. There is no interface 
between domain controllers, but the parent controller has a north-south interface with the domain controller. Cross domain services are computed directly from the parent controller.

This network architecture can form the whole network visualization and virtual logical topology, realize the flexible adjustment of the logical topology, and have the ability to achieve end to end service, channel configuration, routing policy configuration and SLA management in the whole network.

\section{the Key Technology}

In this paper, the key technology of SDN application in the new generation of power dispatch data network is analysed from the perspective of business.

\section{Typical application scenarios of power dispatching based on SDN.}

At present, the dispatching data network provide two VPN for scheduling service, namely real-time VPN and non-real-time VPN, respectively carry safety area I service and security safety area II service. Security area I service mainly includes remote information exchange between EMS system and RTU, power plant automation system of real-time data communication, real-time data exchange between the EMS, data acquisition of the wide area phasor measurement system (WAMS), stability control system, etc. Security area II service mainly includes the contact line exchange plan, the contact line assessment, electric energy measurement information, GPS power station unified clock system data, DTS anti-accident system data, etc.

Analysis of the types and characteristics of service flow in dispatching data network, then according to these characteristics, analysis typical application scenarios of power dispatching based on SDN. On the basis of this research, the network mode and construction mode of power dispatching data network based on SDN and the application network architecture for future electric power dispatching are studied.

\section{North direction interface technology for SDN network service oriented to dispatching system.}

SDN controller provides open north direction interface to service plane and management plane. From the aspects of business, according to the characteristics of the future electric power dispatching, analysis of application requirements such as data acquisition, distributed SCADA, analysis and decision center. Studies the underlying API based on the more fine granularity and SDN north interface standards that is suitable for the substation network access, AGC/AVC control information interaction in analysis and decision center. From the aspects of management, study the specific content of the network service, network scheduling and service management function for the power dispatching application provided by the north interface. Study how to know the working status of network resources and the methods for scheduling network resources.

\section{SDN network configuration technology oriented to dispatching system.}

In view of the requirement of hierarchical and regional control in power dispatching system, need to research effective method for flow table item, which can process data forwarding by using the rules and algorithms of multiple network layers; Design flow chart suitable for electric power dispatching system; Research setting parameters methods about network data path that can secure the real time data of all levels of control center; Study on priority of traffic flow in power dispatching system; Research on real-time data and non-real-time data forwarding strategy. 


\section{Scheduling system reliability of the control information transmission technology based on SDN.}

Traditional networks cannot be centralized to custom end-to-end QoS strategy for a specific business flow, to ensure the important information transmission. How to guarantee the reliable transmission of control information, it is necessary to research the characteristic of the important control information from the integration decision center, such as AGC/AVC message; Research method for identifying control information of dispatching system in SDN network; Research on fine flow scheduling mechanism, design QoS security mechanism for control information; Research on the technology of performance protection and fault self-healing for control information when the link is fault or congested; Research on the solution of single point failure of the controller.

\section{Experimental Verification}

This paper builds a simulation of dispatching data network experiment environment, joining SDN switches in the access network and backbone network edge, which test the convenience of SDN technology to QoS security and the mechanism of automatic routing based on real-time network traffic. Experimental Verification Network topology graph is shown in Fig. 3. SW1, SW2 and SW3 in Fig. 3 are SDN switches, R1-R5 are 5 traditional routers. Two experiments were done, they are respectively the switching path according to the real-time network status and QoS guarantee .

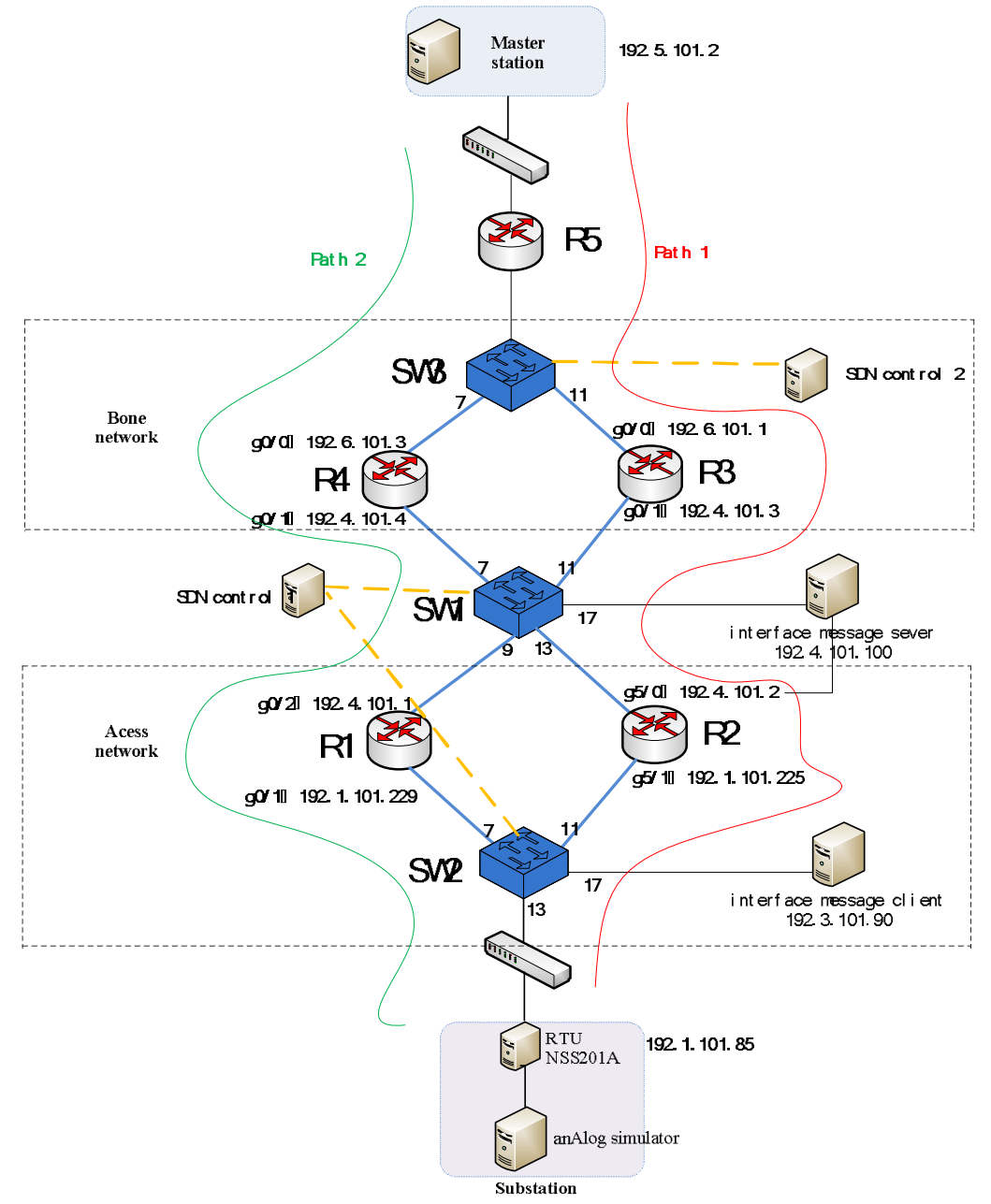

Fig. 3 Experimental Verification Network Topology

\section{The first experiment: according to the network congestion situation, auto select of the relevant path switching.}

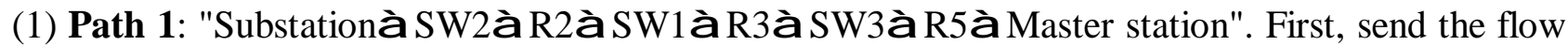
table to path 1 . The other paths have no flow table, so substation messages are transmitted to the 
master station along path 1 . Then refresh the analog 300 signal in the substation, you can see the master station can normally receive the messages transmitted from the substation.

(2) Add the disturbing package: Add the disturbing package from the client to the server with iperf. Here, the disturbing package and the interaction message between master station and substation are along the same path, then you will see the signal immediately refresh slowly, not even refresh.

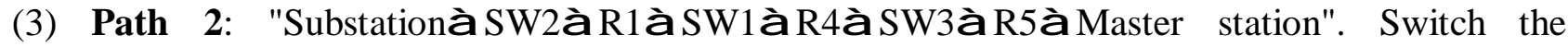
communication path of the substation and master to path 2 , then the master can fluently receive messages transmitted from the substation.

\section{The second experiment: QoS guarantee for the important message.}

(1) Limiting SW1 switch exit speed is 10Mbps.

(2) Set QoS guarantee for the IEC 104 message between substation and the master station.

(3) Using iperf to send ordinary message $10 \mathrm{M}$ / seconds filled SW1 communication port bandwidth, then send different packet size and different rate of 104 packets, the package size is respectively $32 \mathrm{~K}$, $64 \mathrm{~K}, 1 \mathrm{M}$, and the rate is 1 package/10ms, package/100ms, package/s, and observe whether the 104 messages can be received timely and efficiently.

Test results are shown in Table 1.

Table 1. Test results of QoS for important messages based on SDN technology

\begin{tabular}{|c|c|c|c|c|}
\hline $\begin{array}{c}\text { package } \\
\text { size of } \\
\text { IEC 104 } \\
\text { message }\end{array}$ & $\begin{array}{l}\text { 104 message } \\
\text { message } \\
\text { type }\end{array}$ & $\begin{array}{c}\text { send rate of IEC } \\
\text { 1package/ } \\
\text { 10msec }\end{array}$ & $\begin{array}{c}\text { 1 package /100 } \\
\text { msec }\end{array}$ & $\begin{array}{c}\text { 1 package } \\
\text { /1 sec }\end{array}$ \\
\hline \multirow{2}{*}{$32 \mathrm{~K}$} & IEC 104 messages & $0.04 \mathrm{sec}$ & $0.04 \mathrm{sec}$ & $0.04 \mathrm{sec}$ \\
\cline { 2 - 5 } & general messages & overtime & $11.2 \mathrm{sec}$ & $9.2 \mathrm{sec}$ \\
\hline \multirow{2}{*}{$64 \mathrm{~K}$} & IEC 104 messages & $0.07 \mathrm{sec}$ & $0.07 \mathrm{sec}$ & $0.07 \mathrm{sec}$ \\
\cline { 2 - 5 } & general messages & overtime & $13.3 \mathrm{sec}$ & $9.2 \mathrm{sec}$ \\
\hline \multirow{2}{*}{$1 \mathrm{M}$} & IEC 104 messages & $1.07 \mathrm{sec}$ & $1.07 \mathrm{sec}$ & $1.07 \mathrm{sec}$ \\
\cline { 2 - 5 } & general messages & overtime & overtime & $15.17 \mathrm{sec}$ \\
\hline
\end{tabular}

Through the above 2 experiments, SDN technology can be well applied in the power dispatching data network, can automatically select the optimal path according to the network condition, can be more fine-grained to distinguish the relevant message, and can easily set QoS to guarantee the priority of the important message. Through the refinement of the quality control, greatly improve the utilization efficiency of network bandwidth resource, and enhance the flexibility of business deployment.

\section{Conclusion}

SDN technology can provide a panoramic view of the network master station and substation, effectively reduce the complexity of network management, accelerate the network deployment of substation expansion operation and reduce the operation cost of substation network. At the same time, SDN technology provides a wealth of testing means for network quality, and can fast locate fault and reduce network operation error probability, significantly reduce the maintenance cost of substation network. This paper analyses the demand for SDN network dispatching data from a business point of view, discusses the application of the key technology in the new generation power dispatching data network, set up the experimental verification environment to verify the adaptability of SDN technology, provides some useful reference for the unified resource control, network and data collaboration scheduling and quick response to business requirements in new generation of power dispatching data network. Follow up we will continue to deepen the study on the collaborative analysis of operation 
state of scheduling service and running state of load bearing network, realize the early warning ability of the communication network operation state to the dispatching service and improve the reliability of the dispatching business.

\section{Acknowledgements}

This work was financially supported by State Grid Corporation of China (DZ71-16-030).

\section{References}

[1] Mc Keown N 2009 Software-Defined networking. In: Proc. of the INFOCOM Key Note. http://infocom2009.ieee-infocom.org/ technical Program.htm.

[2] Farhady H, Lee H , Nakao A 2015 Software-Defined Networking: A survey . Computer Networks, vol 81 pp 79-95.

[3] Nunes BAA, Mendonca M, Nguyen XN, Obraczka K, Turletti T 2014 A survey of software-defined networking: Past, present, and future of programmable networks. IEEE Communications Surveys and Tutorials, vol 16(3) pp 1617-1634. [doi: 10.1109/SURV.2014. 012214.00180]

[4] ZHANG Chao-Kun, CUI Yong, TANG He-Yi, et al 2015 State-of-the-Art Survey on Software-Defined Networking (SDN) . Journal of Software, vol 26(1) pp 62-81.

[5] HAO Han-yong,HU Zi-wei, LI Jian-qi 2016 Investigation on Typical Applications of SDN in Next Generation Electric Communication Network. Electric Power Information Technology, vol 14(5) pp 65-68.

[6] WU Peng, WU Jun-min, LIU Chuan, et al 2014 Research on the application of software defined network in electric power data communication network. Information Technology, $\operatorname{vol}(1)$ pp 52-55.

[7] QIAO Lin, YIN Xiao-hua, DI Zhuo, et al 2015 Research on SDN network architecture for electric power big data platform. Electric Power Information and Communication Technology, vol 13(1) pp 21-26.

[8] PENG Qingqing, XIANG Li, LU Changyan, et al 2004 Research on power dispatching data network of SGCC. Automtaion of Electric Power System , vol 28(8) pp 10-14.

[9] WANG Yi-min 2005 Design and implementation of state grid dispatching digital network. Power System Technology, vol 29(22) pp 1-6.

[10] YAO Jianguo, YANG Shengchun, DAN Maohua 2013 Reflections on operation supporting system architecture for future interconnected power grid. Power System Technology vol 37(21) pp 52-59.

[11] YAO Jianguo, GAO Zhiyuan, YANG Shengchun 2015 Understanding and prospects of energy internet. Power System Technology vol 39(23) pp 9-14.

[12] XIN Yaozhong, SHI Junjie , ZHOU Jingyang, et al 2015, Technology development trends of smart grid dispatching and control systems . Automation of Electric Power Systems, vol 39( 1) pp $2-8$.

[13] DAN Maohua, YAO Jianguo, YANG Shengchun 2016 Study on Architecture of New Generation of Dispatching Technical Supporting System for Smart Grid . Southern Power System Technology, vol 10(6) pp 1-7. 